\title{
Artificial intelligence methodologies applied to quality control of the positioning services offered by the Red Andaluza de Posicionamiento (RAP) network
}

\author{
Elena Giménez, Antonio José Gil, Department of Cartographic, Geodetic Engineering and \\ Photogrammetry, University of Jaén, Jaén, Spain
}

\begin{abstract}
On April 26, 2012, Elena Giménez de Ory defended her Ph.D. thesis at University of Jaén, entitled: "Robust methodologies applied to quality control of the positioning services offered by the Red Andaluza de Posicionamiento (RAP) network". Elena Giménez de Ory defended her dissertation in a publicly open presentation held in the Higher Polytechnic School at the University of Jaén, and was able to comment on every question raised by her thesis committee and the audience. The thesis was supervised by her advisor, Prof. Antonio J. Gil Cruz, and the rest of his thesis committee, Prof. Manuel Sánchez de la Orden, Dr. Antonio Miguel Ruiz Armenteros and Dr. Gracia Rodríguez Caderot. The thesis has been read and approved by his thesis committee, receiving the highest rating. All of them were present at the presentation.
\end{abstract}

Keywords - RTK, Active GNSS network, Robust Statistics, RAP, Reference frame

\section{THESIS SUMMARY}

$\mathrm{T}$ he development of accurate GNSS (Global Navigation Satellite System) navigation techniques in the recent years, particularly GPS (Global Positioning System), has provided a very important contribution in civil applications: intelligent fleet management systems, social networking, tourist information, traffic management, air traffic control, etc. That is why RTK (Real Time Kinematic) positioning is widely used throughout the world. This is due to the fact that choosing between working at real-time and obtaining centimeter accuracy is no longer necessary: using RTK positioning is possible to combine both. In addition, RTK networks materialize and transmit a reference frame to the users. For this reason, in Andalusia, Spain, an active GNSS network has been materialized, Red Andaluza de Posicionamiento (RAP) [4]. Accuracy studies, implementation of working methodologies and the analysis of the goodness of the obtained results are widely studied topics in Geodesy and Cartographic Sciences [8]. Therefore it is necessary to perform, a rigorous analysis of the two main aspects of these networks: real-time positioning and reference frame. Since statistics techiniques are widely used in artificial intelligence [7], robust methodologies are employed in both analysis methods.

As done in an increasing number of studies, robust methodologies were employed in order to analyze the quality of the real-time positioning services, with particular respect to outlier identification and accuracy assessment. Classical statistical methods do not appear adequate because they are mainly based on the hypothesis of normally distributed samples on Least Squares adjustment results; besides, most outlier detection tests are set up for univariate samples. Thus, a method based on the robust computation of Mahalanobis distances was developed, tested and applied to the RAP Network. This method is able to detect outliers in multivariate samples, as proved by its evaluation by comparing the results obtained from randomly generated data with those stemming from other classical methods.

Moreover, the proposed method is effective with multivariate and small samples, without making assumptions about its distribution, so it can be applied in other problems, no necessary within the field of Geodesy [5].

The application of this method in the processing of RTK positions, recorded in real time with a GNSS receiver assisted by the RAP network, for positioning services is detailed shown in the thesis. Besides, parameters like the coverage of the service, the time to fix ambiguities, and the accuracy and precision of the RTK positioning are also analyzed as described in [6].

In order to analyze the reference frame of the RAP, let us note that the reference frame of a GNSS active network is disseminated to users. But the stations of these networks are not static: due to geodynamic phenomena and local processes change their positions. Thus, the correct realization of the reference frame, as well as the study of the position changes in its permanent stations, are crucial issues in the man-agement of RTK networks.

In Spain, the official reference frame is a realization of the European Terrestrial Reference System 1989 (ETRS89), materialized by the REGENTE network. But Andalusia is 
located along the contact between the Eurasian and African plates, so some RAP permanent station coordinates are not expected to maintain consistency over time with ERTF89. That is why the analysis of the reference frame becomes more necessary.

First, the daily time series solutions must be computed. Processing was performed with the Bernese 5.0 software [3] using the precise ephemerides available from the International GNSS Service (IGS) and absolute calibrated antenna offset values provided by the U.S. National Geodetic Survey (NGS).

The reference frame study has been performed as described in [1]: possible outliers are removed from the time series using LTS (Least Trimmed Squared) method, and using a stochastic model for a signal, the correlated signal can be separated from the uncorrelated noise. Finally, the coordinates were computed by least-squares.

In order to compare the calculated coordinates with the official coordinates of the permanent stations, they must be given in the same reference frame. If permanent station velocities are provided, the transformation can be computed following Boucher and Altamimi [2]. When these velocities are not supplied, a 7-parameter transformation can be computed using, for instance, EUREF permanent stations.

The obtained results show the evolution of the RAP permanent stations positions. The coordinate transformation between official coordinates and calculated coordinates cannot be described as a rigid motion, as expected considering the mentioned geodynamic phenomena.

Finally, the methods described in this thesis, meant to analyze the positioning services of a GNSS active network, can be used to study the positioning services provided by any GNSS active network.

\section{ACKNOWLEDGMENT}

Elena Giménez de Ory received a 4-year $\mathrm{PhD}$ grant from the Spanish Ministry of Education and Science in the period September 2007-August 2011. The research was financed by the research project ESP2006-10113 supported by the Spanish Ministry of Education and Science (European Regional Development Fund-ERDF).

\section{REFERENCES}

[1] Y. Leung, J.H. Ma and W.X. Zhang, "A new method for mining regression classes in large data sets". IEEE Trans. Pattern Analysis Machine Intell., vol 23, pp. 5-21, Jan 2001.
[2] W. Lorenzo, R. González-Crespo and A. Castillo-Sanz, "A Prototype for Linear Features Generalization”. Int. J. Interact. Multimed. Artif. Intell., vol 1, pp. 59-65, Dec 2010.

[3] E. Giménez, M.S. Garrido, M.C. Lacy and A.J. Gil, "Comparing RTK Positioning from Updated REGAM and MERISTEMUM CORS Networks in Southeast Spain", J. Appl. Geodesy, vol 5, pp. 23-35, Apr 2011.

[4] B. Benciolini, L. Biagi, M. Crespi, A. M. Manzino, and M. Roggero, "Reference frames for GNSS positioning services: Some problems and proposed solutions", J.Appl.Geodesy, vol 2, pp. 53-62, Apr 2008

[5] M.S. Garrido, E. Giménez, J.A. Armenteros, M. C. Lacy and A.J. Gil, "Evaluation of NRTK Positioning Using the RENEP and RAP Networks on the Southern Border Region of Portugal and Spain", Acta Geod. Geophys. Hung., vol 47, pp. 52-65, Mar 2012.

[6] E. Giménez, M. Crespi, M.S. Garrido and A.J. Gil, "Multivariate Outlier Detection Based on Robust Computation of Mahalanobis Distances, Application to Positioning Assisted by RTK GNSS Networks". Int. J. Appl Earth Obs. Geoinf. 16, pp. 94-100, Jun 2012.

[7] Bernese GPS Software Version 5.0, University of Bern, Bern, Switzerland, 2006.

[8] C. Boucher. and Z. Altamimi (May 2011). Memo: Specifications for reference frame fixing in the analysis of a EUREF GPS campaign. Available: http://etrs89.ensg.ign.fr/memo-V8.pdf.

Elena Giménez de Ory is an Associate Professor at the Pontifical University of Salamanca. On 2012 she defended her Ph.D. thesis in Geodetic and Cartographic Engineering at the University of Jaén. Her current research interests include robust statistics and its applications to RTK positioning based on active GNSS networks.

Antonio J. Gil is a full professor of Geodesy at the department of Cartographic, Geodetic and Photogrammetric Engineering at the University of Jaén. He has been the Head of the UJA Geodesy Research Group since 1997. His current research interests include, among others, Global Navigation Satellite Systems and positioning and navigation based on active GNSS networks. He is author of more than a hundred works, articles and conference reports. 